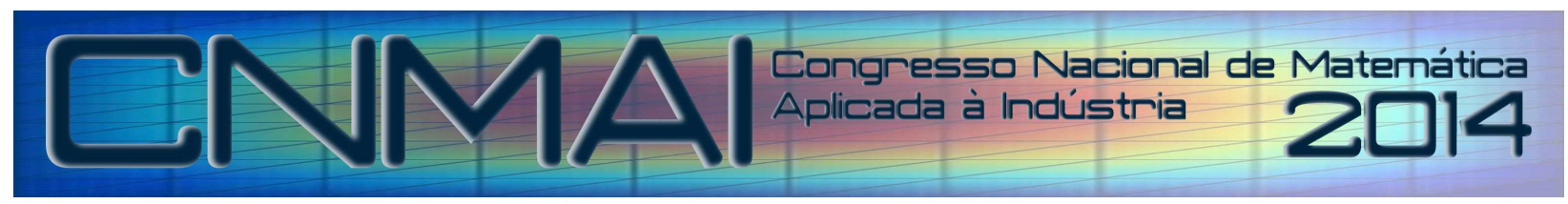

18 a 21 de novembro de 2014, Caldas Novas - Goiás

\title{
OTIMIZAÇÃO DOS CICLOS DE AR-PADRÃO OTTO, DIESEL E DUAL COM RELAÇÃO A POTÊNCIA LÍQUIDA DE SAÍDA E A PRESSÃO MÉDIA EFETIVA
}

\author{
Santiago del Rio Oliveira, santiago@ feb.unesp.br ${ }^{1}$ \\ Vicente Luiz Scalon, scalon@feb.unesp.br ${ }^{1}$ \\ ${ }^{1}$ Universidade Estadual Paulista Júlio de Mesquita Filho/UNESP - Departamento de Engenharia Mecânica - Av. Eng. \\ Luiz Edmundo C. Coube, Bairro Vargem Limpa, CEP 17033-360, Bauru/SP.
}

\begin{abstract}
Resumo: Nesse trabalho os ciclos endoreversíveis de ar-padrão Otto, Diesel e Dual são otimizados com relação a potência líquida de saída e a pressão média efetiva. O ciclo endoreversível é aquele em que o processo de fornecimento de calor a partir da combustão e o processo de rejeição de calor para o ambiente são os únicos processos irreversíveis no ciclo. São obtidas expressões para a potência líquida de saída otimizada e para a pressão média efetiva otimizada para cada ciclo motor e é realizada então uma análise comparativa dos resultados obtidos. Estes resultados podem ser utilizados como um critério adicional para uso na avaliação de desempenho e adequação de motores de combustão interna que operam conforme os ciclos Otto, Diesel e Dual.
\end{abstract}

Palavras-chave: Otto, Diesel, Dual, otimização

\section{INTRODUÇÃO}

Máquinas térmicas a vapor foram desenvolvidas no século 18 para fornecer a humanidade meios de fornecimento de trabalho sob demanda. Entretanto, as dimensões e os altos custos de operação associados às máquinas a vapor incentivaram diversos pesquisadores a procurar por alternativas mais viáveis. Uma das idéias inovadoras foi substituir o vapor por ar como fluido de trabalho de maneira a permitir a utilização da combustão interna ao próprio cilindro. Isso evitaria a fornalha, a caldeira e o sistema de tubulações necessários aos sistemas de produção de vapor.

Foi o engenheiro alemão Nikolaus Otto, em 1876, quem conseguiu de fato construir o motor de quatro tempos, consolidando a hipótese de que a fase de compressão seria responsável por um considerável aumento de potência do motor. A patente deste motor foi dada a Otto, o que tornou este ciclo conhecido como ciclo Otto. Em fevereiro de 1897 , o engenheiro alemão Rudolf Diesel fez o pedido de patente de um motor que possuía alta compressão e grande economia de combustível. Com a patente concedida este ciclo ficou conhecido com ciclo Diesel. Proposto como uma combinação do ciclo Otto e ciclo Diesel, o ciclo Dual visa dispor um maior tempo para queima de combustíveis sendo amplamente utilizado em motores à diesel.

Atualmente, práticas de engenharia fazem uso dos ciclos de ar-padrão Otto, Diesel e Dual para fornecer uma breve descrição dos motores Otto, Diesel e Dual (Chen, et al., 1998 e Chen, et al., 1996). Essas práticas têm vantagens e desvantagens. Resultados apresentados por uma análise de ar-padrão são indicadores úteis de tendências a serem seguidas por uma ou mais variáveis dos motores. O objetivo da formulação desse trabalho não é ser qualitativa, mas de fornecer um melhor entendimento das tendências relacionadas à otimização da potência líquida de saída e da pressão média efetiva dos ciclos de ar-padrão Otto, Diesel e Dual.

\section{MODELO MATEMÁTICO}

Para os ciclos analisados, os seguintes símbolos são utilizados: $r$ é a razão de compressão, $r_{c}$ é a razão de corte, $r_{p}$ é a razão de pressão, $x$ é a relação entre temperaturas, $\dot{W}_{\text {ciclo }}$ é a potência líquida de saída, $P M E$ é a pressão média efetiva, $\eta$ é a eficiência térmica, $u$ é a energia interna específica, $\dot{m}$ é a vazão mássica do fluido de trabalho no ciclo, 
$c_{v}$ é o calor específico a volume constante, $c_{p}$ é o calor específico a pressão constante, $V$ é o volume, $T$ é a temperatura e $k$ é a relação entre calores específicos. Além disso, os termos "ponto morto superior" e "ponto morto inferior" serão reescritos por simplicidade como PMS e PMI, respectivamente.

\subsection{Ciclo Otto}

O ciclo de ar-padrão Otto é um ciclo ideal que considera que a adição de calor ocorre instantaneamente enquanto o pistão encontra-se no PMS. O ciclo Otto é mostrado nos diagramas $p-v$ e $T-s$ da Fig. 1 . O ciclo consiste em quatro processos internamente reversíveis em série. Segue abaixo uma breve descrição de cada um dos quatro processos:

- PROCESSO 1-2: trabalho de compressão isoentrópico do ar $W_{12}$ conforme o pistão se move do PMI para o PMS.

- PROCESSO 2-3: transferência de calor a volume constante para o ar $Q_{23}$ a partir de uma fonte externa enquanto o pistão está no PMS.

- PROCESSO 3-4: trabalho de expansão isoentrópico do ar $W_{34}$ conforme o pistão se move do PMS para o PMI.

- PROCESSO 4-1: transferência de calor a volume constante do ar $Q_{41}$ enquanto o pistão está no PMI.

Para esse ciclo idealizado, a alteração de composição química relacionada à combustão foi desprezada. A temperatura ao final da combustão, $T_{3}$, depende da taxa de combustão $\dot{Q}_{23}$. Quanto mais alta for a taxa de combustão por uma dimensão fixa de cilindro e razão de compressão, maior a temperatura $T_{3}$ ao final da combustão. Como $T_{3}$ é a temperatura mais alta e $T_{1}$ a temperatura mais baixa, espera-se que a eficiência resultante seja menor do que a eficiência de Carnot operando entre essas duas temperaturas extremas.

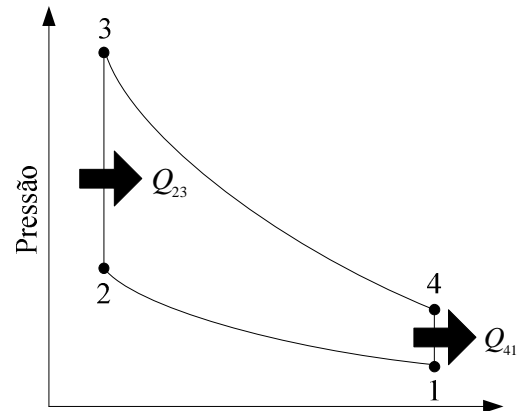

Volume

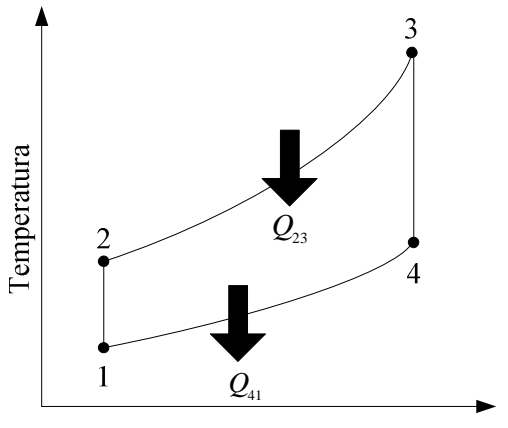

Entropia

Figura 1. Diagramas $p-v$ e $T-s$ do ciclo de ar-padrão Otto.

\subsubsection{Formulação}

De acordo a Fig. 1 e utilizando a hipótese de ar-padrão frio numa base temporal, obtêm-se as seguintes expressões:

$$
\begin{aligned}
& \dot{W}_{12}=\dot{m}\left(u_{2}-u_{1}\right)=\dot{m} c_{v}\left(T_{2}-T_{1}\right) \\
& \dot{W}_{34}=\dot{m}\left(u_{3}-u_{4}\right)=\dot{m} c_{v}\left(T_{3}-T_{4}\right) \\
& \dot{Q}_{23}=\dot{m}\left(u_{3}-u_{2}\right)=\dot{m} c_{v}\left(T_{3}-T_{2}\right) \\
& r=V_{1} / V_{2}=V_{4} / V_{3} \\
& x=T_{2} / T_{1}=r^{k-1}=T_{3} / T_{4} \\
& \dot{W}_{\text {ciclo, Otto }}=\dot{W}_{34}-\dot{W}_{12}=\dot{m} c_{v}\left[T_{3}(1-1 / x)-T_{1}(x-1)\right] \\
& P M E_{\text {Otto }}=\frac{\dot{W}_{\text {ciclo,otoo }}}{V_{1}-V_{2}}=\frac{\dot{m} c_{v}}{V_{1}} \times \frac{T_{3}(1-1 / x)-T_{1}(x-1)}{1-x^{1 /(1-k)}} \\
& \eta_{\text {Oto }}=\frac{\dot{W}_{\text {ciclo, Otoo }}}{\dot{Q}_{23}}=\frac{\dot{m} c_{v}\left(T_{3}-T_{4}\right)-\dot{m} c_{v}\left(T_{2}-T_{1}\right)}{\dot{m} c_{v}\left(T_{3}-T_{2}\right)}=1-\frac{1}{r^{k-1}}
\end{aligned}
$$




\subsubsection{Otimização da potência líquida de saída}

Como pode ser visto pela Eq. (6), $\dot{W}_{\text {ciclo.oto }}$ é função somente da relação de temperaturas $x$. A otimização da potência líquida de saída pode ser realizada através da diferenciação da Eq. (6) com relação a $x$ e posterior verificação do comportamento da função utilizando o critério da segunda derivada. As derivadas são escritas como:

$$
\begin{gathered}
\frac{d \dot{W}_{\text {ciclo, Oto }}}{d x}=\frac{d}{d x}\left\{\dot{m} c_{v}\left[T_{3}\left(1-\frac{1}{x}\right)-T_{1}(x-1)\right]\right\}=\dot{m} c_{v}\left(T_{3} x^{-2}-T_{1}\right) \\
\frac{d^{2} \dot{W}_{\text {ciclo.Oto }}}{d x^{2}}=\frac{d}{d x}\left[\frac{d \dot{W}_{\text {ciclo.Oto }}}{d x}\right]=\frac{d}{d x}\left[\dot{m} c_{v}\left(T_{3} x^{-2}-T_{1}\right)\right]=-2 T_{3} \dot{m} c_{v} x^{-3}
\end{gathered}
$$

Igualando a Eq. (9) a zero, obtém-se um ponto crítico e a substituição desse ponto crítico na Eq. (10) indica se o ponto crítico maximiza ou minimiza a potência líquida de saída. Após essas operaçãos obtém-se:

$$
\begin{gathered}
x_{\text {cri }}=\sqrt{T_{3} / T_{1}} \\
\left.\frac{d^{2} \dot{W}_{\text {ciclo Ooto }}}{d x^{2}}\right|_{x_{\text {ci }}}=-2 T_{3} \dot{m} c_{v}\left(\sqrt{T_{3} / T_{1}}\right)^{-3}<0
\end{gathered}
$$

De acordo com a Eq. (12), o ponto crítico maximiza a potência líquida de saída. Substituindo a Eq. (11) na Eq. (6) obtém-se a potência líquida de saída otimizada e substituindo a Eq. (12) nas Eqs. (5), (7) e (8) obtém-se a razão de compressão otimizada, a pressão média efetiva otimizada e a eficiência térmica otimizada, respectivamente, ou seja:

$$
\begin{gathered}
\left(\dot{W}_{\text {ciclo, Oto }}\right)_{\text {otm }}=\dot{m} c_{v}\left(\sqrt{T_{3}}-\sqrt{T_{1}}\right)^{2} \\
r_{\text {otm }, \text { otto }}=\left(T_{3} / T_{1}\right)^{\frac{1}{2(k-1)}} \\
P M E_{\text {otm, Oto }}=\frac{\dot{m} c_{v}}{V_{1}} \times \frac{\left(\sqrt{T_{3}}-\sqrt{T_{1}}\right)^{2}}{1-\left(\sqrt{T_{3} / T_{1}}\right)^{1 /(1-k)}} \\
\eta_{\text {otm,Oto }}=1-\sqrt{T_{1} / T_{3}}
\end{gathered}
$$

\subsubsection{Otimização da pressão média efetiva}

A PME, de acordo com a Eq. (7), também é função de somente da variável $x$ e pode ser maximizada através de:

$$
d(P M E) / d x=0
$$

O resultado da equação acima não pode ser explicitado, sendo representado por uma equação transcendental:

$$
x_{\max }=\left[\frac{\left(T_{3} / x_{\max }^{2}-T_{1}\right)(1-k)\left(1-x_{\max }^{1 /(1-k)}\right)}{T_{1}\left(x_{\max }-1\right)-T_{3}\left(1-1 / x_{\max }\right)}\right]^{(1-k) / k}
$$

Foi verificado computacionalmente que o resultado da Eq. (18) resulta em um máximo da Eq. (7). A Eq. (18) deve ser resolvida numericamente para a obtenção do valor de $x_{\max }$. Com o valor de $x_{\max }$, podem ser calculados valores de $\left(\dot{W}_{\text {ciclo,oto }}\right)_{\max }, r_{\max , \text { Oto }}, P M E_{\max , \text { Oto }}$ e $\eta_{\max , \text { Oto }}$, respectivamente, através das seguintes expressões:

$$
\left(\dot{W}_{\text {ciclo, Oto }}\right)_{\max }=\dot{m} c_{v}\left[T_{3}\left(1-1 / x_{\max }\right)-T_{1}\left(x_{\max }-1\right)\right]
$$




$$
\begin{gathered}
r_{\text {max }, \text { Otto }}=\left(x_{\max }\right)^{1 /(1-k)} \\
P M E_{\text {max }, \text { oto }}=\frac{\dot{m} c_{v}}{V_{1}} \times \frac{T_{3}\left(1-1 / x_{\max }\right)-T_{1}\left(x_{\max }-1\right)}{1-x_{\max }^{1 /(1-k)}} \\
\eta_{\max , \text { Oto }}=\frac{T_{3}\left(1-1 / x_{\max }\right)-T_{2}\left(x_{\max }-1\right)}{T_{3}-T_{1} x_{\max }}
\end{gathered}
$$

\subsection{Ciclo Diesel}

O ciclo de ar-padrão Diesel é um ciclo ideal que considera que a adição de calor ocorre durante um processo a pressão constante, quando o pistão se desloca do PMS para o PMI. O ciclo Diesel é mostrado nos diagramas $p-v$ e $T-s$ da Fig. 2. O ciclo consiste em quatro processos internamente reversíveis em série. Segue abaixo uma breve descrição de cada um dos quatro processos:

- PROCESSO 1-2: trabalho de compressão isoentrópico do ar $W_{12}$ conforme o pistão se move do PMI para o PMS.

- PROCESSO 2-3: transferência de calor a pressão constante para o ar $Q_{23}$ a partir de uma fonte externa (1 ${ }^{\mathrm{a}}$ parte do curso de potência).

- PROCESSO 3-4: trabalho de expansão isoentrópico do ar $W_{34}$ conforme o pistão se move para o PMI.

- PROCESSO 4-1: transferência de calor a volume constante do ar $Q_{41}$ enquanto o pistão está no PMI.

Da mesma forma que no ciclo Otto, para esse ciclo idealizado, a alteração de composição química relacionada a combustão foi desprezada. A temperatura ao final da combustão, $T_{3}$, depende da taxa de combustão $\dot{Q}_{23}$. Quanto mais alta for a taxa de combustão por uma dimensão fixa de cilindro e razão de compressão, maior a temperatura $T_{3}$ ao final da combustão. Como $T_{3}$ é a temperatura mais alta e $T_{1}$ a temperatura mais baixa, espera-se que a eficiência resultante seja menor do que a eficiência de Carnot operando entre essas duas temperaturas extremas.
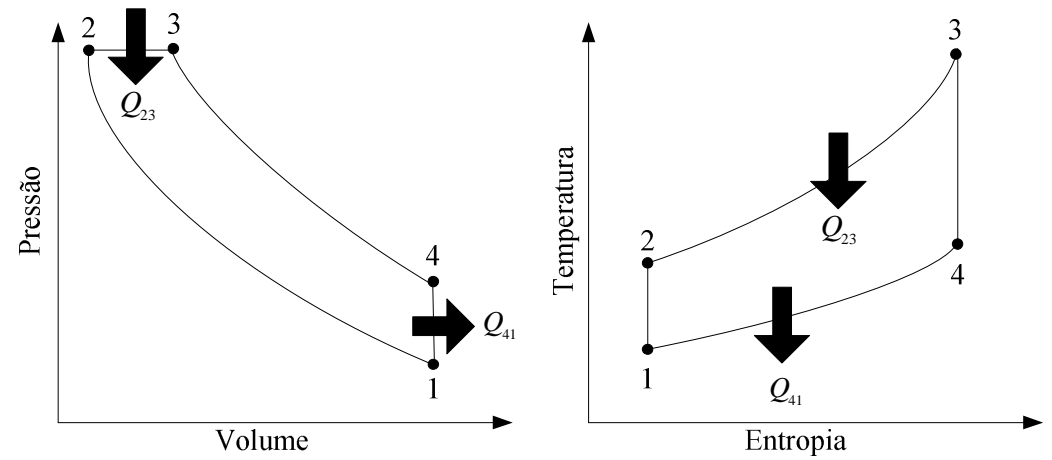

Figura 2. Diagramas $p-v$ e $T-s$ do ciclo de ar-padrão Diesel.

\subsubsection{Formulação}

De acordo a Fig. 2 e utilizando a hipótese de ar-padrão frio numa base temporal, obtêm-se as seguintes expressões:

$$
\begin{gathered}
\dot{W}_{12}=\dot{m}\left(u_{2}-u_{1}\right)=\dot{m} c_{v}\left(T_{2}-T_{1}\right) \\
\dot{W}_{23}=\dot{m}\left(h_{3}-h_{2}\right)-\dot{m}\left(u_{3}-u_{2}\right)=\dot{m} c_{p}\left(T_{3}-T_{2}\right)-\dot{m} c_{v}\left(T_{3}-T_{2}\right) \\
\dot{W}_{34}=\dot{m}\left(u_{3}-u_{4}\right)=\dot{m} c_{v}\left(T_{3}-T_{4}\right) \\
\dot{Q}_{23}=\dot{m}\left(h_{3}-h_{2}\right)=\dot{m} c_{p}\left(T_{3}-T_{2}\right) \\
r=V_{1} / V_{2}=V_{4} / V_{2} \\
r_{c}=V_{3} / V_{2}
\end{gathered}
$$




$$
\begin{gathered}
x=T_{2} / T_{1}=r^{k-1} \\
x / r_{c}^{k-1}=T_{3} / T_{4} \\
\dot{W}_{\text {ciclo, Diesel }}=\dot{W}_{23}+\dot{W}_{34}-\dot{W}_{12}=\dot{m} c_{v}\left[T_{3}\left(1-r_{c}^{k-1} / x\right)+(k-1) T_{3}\left(1-1 / r_{c}\right)-T_{1}(x-1)\right] \\
P M E_{\text {Diesel }}=\frac{\dot{W}_{\text {ciclo, Diesel }}}{V_{1}-V_{2}}=\frac{\dot{m} c_{v}}{V_{1}} \times \frac{\left[T_{3}\left(1-r_{c}^{k-1} / x\right)+(k-1) T_{3}\left(1-1 / r_{c}\right)-T_{1}(x-1)\right]}{1-x^{1 /(1-k)}} \\
\dot{\eta}_{\text {Diesel }}=\frac{\dot{W}_{\text {ciclo, Dissel }}}{\dot{Q}_{23}}=\frac{\dot{m} c_{p}\left(T_{3}-T_{2}\right)-\dot{m} c_{v}\left(T_{3}-T_{2}\right)+\dot{m} c_{v}\left(T_{3}-T_{4}\right)-\dot{m} c_{v}\left(T_{2}-T_{1}\right)}{\dot{m} c_{p}\left(T_{3}-T_{2}\right)}=1-\frac{1}{r^{k-1}}\left[\frac{r_{c}^{k}-1}{k\left(r_{c}-1\right)}\right]
\end{gathered}
$$

\subsubsection{Otimização da potência líquida de saída}

Como pode ser visto pela Eq. (31), $\dot{W}_{\text {ciclo, Dises }}$ é função somente da relação de temperaturas $x$. A otimização da potência líquida de saída pode ser realizada através da diferenciação da Eq. (31) com relação a $x$ e posterior verificação do comportamento da função utilizando o critério da segunda derivada. As derivadas são escritas como:

$$
\begin{gathered}
\frac{d \dot{W}_{\text {ciclo.Diesel }}}{d x}=\frac{d}{d x}\left\{\dot{m} c_{v}\left[T_{3}\left(1-r_{c}^{k-1} / x\right)+(k-1) T_{3}\left(1-1 / r_{c}\right)-T_{1}(x-1)\right]\right\}=\dot{m} c_{v}\left(T_{3} r_{c}^{k-1} x^{-2}-T_{1}\right) \\
\frac{d^{2} \dot{W}_{\text {ciclo.Diesel }}}{d x^{2}}=\frac{d}{d x}\left[\frac{d \dot{W}_{\text {ciclo.Diesel }}}{d x}\right]=\frac{d}{d x}\left[\dot{m} c_{v}\left(T_{3} r_{c}^{k-1} x^{-2}-T_{1}\right)\right]=-2 T_{3} r_{c}^{k-1} \dot{m} c_{v} x^{-3}
\end{gathered}
$$

Igualando a Eq. (34) a zero, obtém-se um ponto crítico e a substituição desse ponto crítico na Eq. (35) indica se o ponto crítico maximiza ou minimiza a potência líquida de saída. Após essas operaçãos obtém-se:

$$
\begin{gathered}
x_{c r i}=\sqrt{T_{3} r_{c}^{k-1} / T_{1}} \\
\left.\frac{d^{2} \dot{W}_{\text {ciclo, Disel }}}{d x^{2}}\right|_{x_{c i}}=-2 T_{3} \dot{m} c_{v}\left(\sqrt{T_{3} r_{c}^{k-1} / T_{1}}\right)^{-3}<0
\end{gathered}
$$

De acordo com a Eq. (37), o ponto crítico maximiza a potência líquida de saída. Substituindo a Eq. (36) na Eq. (31) obtém-se a potência líquida de saída otimizada e substituindo a Eq. (36) nas Eqs. (29), (32) e (33) obtém-se a razão de compressão otimizada, a pressão média efetiva otimizada e a eficiência térmica otimizada, respectivamente, ou seja:

$$
\begin{gathered}
\left(\dot{W}_{\text {ciclo,Diesel }}\right)_{\text {otm }}=\dot{m} c_{v}\left[T_{3}-2 \sqrt{T_{1} T_{3} r_{c}^{k-1}}+(k-1) T_{3}\left(1-\frac{1}{r_{c}}\right)-T_{1}\right] \\
r_{r_{\text {otm,Diesel }}}=\left(\frac{T_{3} r_{c}^{k-1}}{T_{1}}\right)^{\frac{1}{2(k-1)}} \\
P M E_{\text {otm, Diesel }}=\frac{\dot{m} c_{v}}{V_{1}} \times \frac{\left[T_{3}-2 \sqrt{T_{1} T_{3} r_{c}^{k-1}}+(k-1) T_{3}\left(1-\frac{1}{r_{c}}\right)-T_{1}\right]}{1-\left(\sqrt{T_{3} r_{c}^{k-1} / T_{1}}\right)^{1 /(1-k)}} \\
\eta_{\text {otm, Diesel }}=1-\sqrt{\frac{T_{1}}{T_{3} r_{c}^{k-1}}\left[\frac{r_{c}^{k}-1}{k\left(r_{c}-1\right)}\right]}
\end{gathered}
$$




\subsubsection{Otimização da pressão média efetiva}

A PME, de acordo com a Eq. (32), também é função de somente da variável $x$ e pode ser maximizada através de:

$$
d(P M E) / d x=0
$$

O resultado da equação acima não pode ser explicitado, sendo representado por uma equação transcendental:

$$
x_{\max }=\left[\frac{\left(T_{3} / x_{\max }^{2}-T_{1}\right)(1-k)\left(1-x_{\max }^{1 /(1-k)}\right)}{T_{1}\left(x_{\max }-1\right)-T_{3}\left(1-1 / x_{\max }\right)+(k-1) T_{3}\left(1-1 / r_{c}\right)}\right]^{(1-k) / k}
$$

Foi verificado computacionalmente que o resultado da Eq. (43) resulta em um máximo da Eq. (32). A Eq. (43) deve ser resolvida numericamente para a obtenção do valor de $x_{\max }$. Com o valor de $x_{\max }$, podem ser calculados valores de $\left(\dot{W}_{\text {ciclo,Diesel }}\right)_{\max }, r_{\max , \text { Diesel }}, P M E_{\text {max,Diesel }}$ e $\eta_{\text {max,Diesel }}$, respectivamente, através das seguintes expressões:

$$
\begin{gathered}
\left(\dot{W}_{\text {ciclo,Diesel }}\right)_{\max }=\dot{m} c_{v}\left[T_{3}\left(1-1 / x_{\max }\right)-T_{1}\left(x_{\max }-1\right)\right] \\
r_{\text {max }, \text { Diesel }}=\left(x_{\max }\right)^{1 /(1-k)} \\
P M E_{\text {max,Diesel }}=\frac{\dot{m} c_{v}}{V_{1}} \times \frac{\left[T_{3}\left(1-r_{c}^{k-1} / x_{\max }\right)+(k-1) T_{3}\left(1-1 / r_{c}\right)-T_{1}\left(x_{\max }-1\right)\right]}{1-x_{\max }^{1 /(1-k)}} \\
\eta_{\text {max,Diesel }}=\frac{T_{3}\left(1-1 / x_{\max }\right)-T_{2}\left(x_{\max }-1\right)}{T_{3}-T_{1} x_{\text {max }}}
\end{gathered}
$$

\subsection{Ciclo Dual}

O ciclo de ar-padrão Dual é um ciclo ideal que considera que a adição de calor ocorre em duas etapas. Na primeira etapa, a adição de calor ocorre instantâneamente enquanto o pistão encontra-se no PMS. Na segunda etapa a adição de calor ocorre durante um processo à pressão constante, quando o pistão se desloca do PMS para o PMI. O ciclo Dual é mostrado nos diagramas $p-v$ e $T-s$ da Fig. 3. O ciclo consiste em cinco processos internamente reversíveis em série. Segue abaixo uma breve descrição de cada um dos cinco processos:

- PROCESSO 1-2: trabalho de compressão isoentrópico do ar $W_{12}$ conforme o pistão se move do PMI para o PMS.

- PROCESSO 2-3: transferência de calor a volume constante para o ar $Q_{23}$ a partir de uma fonte externa enquanto o pistão está no PMS.

- $\quad$ PROCESSO 3-4: transferência de calor a pressão constante para o ar $Q_{34}$ a partir de uma fonte externa (1 ${ }^{\mathrm{a}}$ parte do curso de potência).

- $\quad$ PROCESSO 4-5: potência de expansão isoentrópica do ar $W_{45}$ conforme o pistão se move para o PMI.

- PROCESSO 5-1: taxa de transferência de calor a volume constante do ar $Q_{51}$ enquanto o pistão está no PMI.

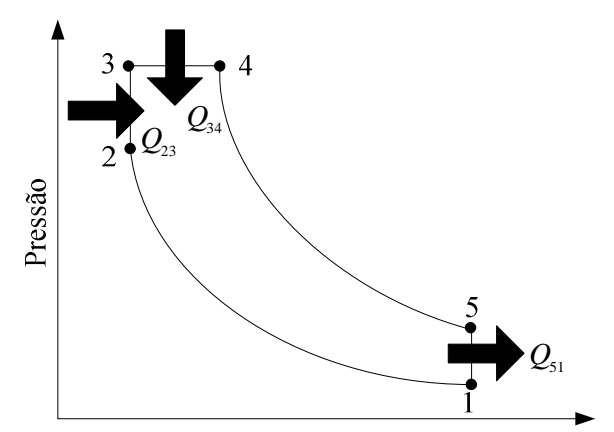

Volume

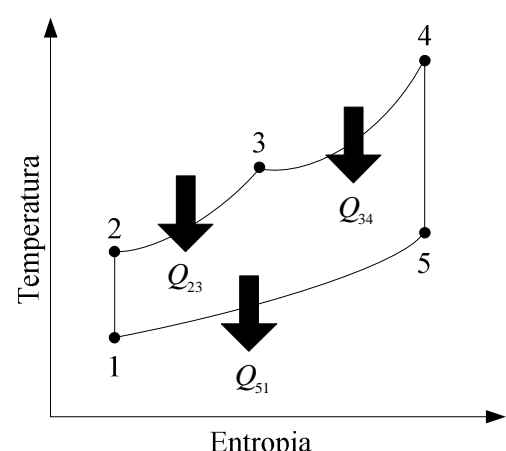

Entropia

Figura 3. Diagramas $p-v$ e $T-s$ do ciclo de ar-padrão Dual.

Da mesma forma que nos ciclos Otto e Diesel, para esse ciclo idealizado, a alteração de composição química relacionada a combustão foi desprezada. A temperatura ao final da combustão, $T_{4}$, depende da taxa de combustão $\dot{Q}_{34}$. 
Quanto mais alta for a taxa de combustão por uma dimensão fixa de cilindro e razão de compressão, maior a temperatura $T_{4}$ ao final da combustão. Como $T_{4}$ é a temperatura mais alta e $T_{1}$ a temperatura mais baixa, espera-se que a eficiência resultante seja menor do que a eficiência de Carnot operando entre essas duas temperaturas extremas.

\subsubsection{Formulação}

De acordo a Fig. 3 e utilizando a hipótese de ar-padrão frio numa base temporal, obtêm-se as seguintes expressões:

$$
\begin{gathered}
\dot{W}_{12}=\dot{m}\left(u_{2}-u_{1}\right)=\dot{m} c_{v}\left(T_{2}-T_{1}\right) \\
\dot{W}_{34}=\dot{m}\left(h_{4}-h_{3}\right)-\dot{m}\left(u_{4}-u_{3}\right)=\dot{m} c_{p}\left(T_{4}-T_{3}\right)-\dot{m} c_{v}\left(T_{4}-T_{3}\right) \\
\dot{W}_{45}=\dot{m}\left(u_{4}-u_{5}\right)=\dot{m} c_{v}\left(T_{4}-T_{5}\right) \\
\dot{Q}_{23}=\dot{m}\left(u_{3}-u_{2}\right)=\dot{m} c_{v}\left(T_{3}-T_{2}\right) \\
\dot{Q}_{34}=\dot{m}\left(h_{4}-h_{3}\right)=\dot{m} c_{p}\left(T_{4}-T_{3}\right) \\
r=V_{1} / V_{2}=V_{5} / V_{3} \\
r_{c}=V_{4} / V_{3} \\
r_{p}=p_{3} / p_{2} \\
x=T_{2} / T_{1}=r^{k-1} \\
x / r_{c}^{k-1}=T_{4} / T_{5} \\
\eta_{\text {Dual }}=\frac{\dot{W}_{\text {ciclo, Dual }}}{\dot{Q}_{23}+\dot{Q}_{34}}=\frac{\dot{m} c_{p}\left(T_{4}-T_{3}\right)-\dot{m} c_{v}\left(T_{4}-T_{3}\right)+\dot{m} c_{v}\left(T_{4}-T_{5}\right)-\dot{m} c_{v}\left(T_{2}-T_{1}\right)}{\dot{m} c_{v}\left(T_{3}-T_{2}\right)+\dot{m} c_{p}\left(T_{4}-T_{3}\right)} \\
\dot{W}_{\text {ciclo, Dual }}=\dot{W}_{34}+\dot{W}_{45}-\dot{W}_{12}= \\
\dot{m} c_{v}\left[T_{4}\left(1-r_{c}^{k-1} / x\right)+(k-1) T_{4}\left(1-1 / r_{c}\right)-T_{1}(x-1)\right] \\
\left.\dot{W}_{\text {ciclo, Dual }}^{k-1}=\frac{\dot{m} c_{v}}{V_{1}} \times \frac{\left[T_{4}\left(1-r_{c}^{k-1} / x\right)+(k-1) T_{4}\left(1-1 / r_{c}\right)-T_{1}(x-1)\right]}{\left(r_{p}-1\right)+k r_{p}\left(r_{c}-1\right)}\right] \\
P M E_{\text {Dual }}=r_{2}^{k}-1
\end{gathered}
$$

\subsubsection{Otimização da potência líquida de saída}

Como pode ser visto pela Eq. (58), $\dot{W}_{\text {ciclo.Dual }}$ é função somente da relação de temperaturas $x$. A otimização da potência líquida de saída pode ser realizada através da diferenciação da Eq. (58) com relação a $x$ e posterior verificação do comportamento da função utilizando o critério da segunda derivada. As derivadas são escritas como:

$$
\begin{gathered}
\frac{d \dot{W}_{\text {ciclo, Dual }}}{d x}=\frac{d}{d x}\left\{\dot{m} c_{v}\left[T_{4}\left(1-\frac{r_{c}^{k-1}}{x}\right)+(k-1) T_{4}\left(1-\frac{1}{r_{c}}\right)-T_{1}(x-1)\right]\right\}=\dot{m} c_{v}\left(T_{4} r_{c}^{k-1} x^{-2}-T_{1}\right) \\
\frac{d^{2} \dot{W}_{\text {ciclo, Dual }}}{d x^{2}}=\frac{d}{d x}\left[\frac{d \dot{W}_{\text {cillo, Dual }}}{d x}\right]=\frac{d}{d x}\left[\dot{m} c_{v}\left(T_{4} r_{c}^{k-1} x^{-2}-T_{1}\right)\right]=-2 T_{4} r_{c}^{k-1} \dot{m} c_{v} x^{-3}
\end{gathered}
$$

Igualando a Eq. (61) a zero, obtém-se um ponto crítico e a substituição desse ponto crítico na Eq. (58) indica se o ponto crítico maximiza ou minimiza a potência líquida de saída. Após essas operaçãos obtém-se:

$$
x_{c r i}=\sqrt{T_{4} r_{c}^{k-1} / T_{1}}
$$




$$
\left.\frac{d^{2} \dot{W}_{\text {ciclo.Dual }}}{d x^{2}}\right|_{x_{c i}}=-2 T_{4} \dot{m} c_{v}\left(\sqrt{T_{4} r_{c}^{k-1} / T_{1}}\right)^{-3}<0
$$

De acordo com a Eq. (64), o ponto crítico maximiza a potência líquida de saída. Substituindo a Eq. (63) na Eq. (58) obtém-se a potência líquida de saída otimizada e substituindo a Eq. (63) nas Eqs. (56), (59) e (60) obtém-se a razão de compressão otimizada, a pressão média efetiva otimizada e a eficiência térmica otimizada, respectivamente, ou seja:

$$
\begin{gathered}
\left(\dot{W}_{\text {ciclo, Dual }}\right)_{\text {otm }}=\dot{m} c_{v}\left[T_{4}-2 \sqrt{T_{1} T_{4} r_{c}^{k-1}}+(k-1) T_{4}\left(1-\frac{1}{r_{c}}\right)-T_{1}\right] \\
r_{\text {otm, Dual }}=\left(\frac{T_{4} r_{c}^{k-1}}{T_{1}}\right)^{\frac{1}{2(k-1)}} \\
P M E_{\text {otm, Dual }}=\frac{\dot{m} c_{v}}{V_{1}} \times \frac{\left[T_{4}-2 \sqrt{T_{1} T_{4} r_{c}^{k-1}}+(k-1) T_{4}\left(1-\frac{1}{r_{c}}\right)-T_{1}\right]}{1-\left(\sqrt{T_{4} r_{c}^{k-1} / T_{1}}\right)^{1 /(1-k)}} \\
\eta_{\text {otm, Dual }}=1-\sqrt{\frac{T_{1}}{T_{4} r_{c}^{k-1}}}\left[\frac{r_{p} r_{c}^{k}-1}{\left(r_{p}-1\right)+k r_{p}\left(r_{c}-1\right)}\right]
\end{gathered}
$$

\subsubsection{Otimização da pressão média efetiva}

A PME, de acordo com a Eq. (59), também é função de somente da variável $x$ e pode ser maximizada através de:

$$
d(P M E) / d x=0
$$

O resultado da equação acima não pode ser explicitado, sendo representado por uma equação transcendental

$$
x_{\max }=\left[\frac{\left(T_{4} / x_{\max }^{2}-T_{1}\right)(1-k)\left(1-x_{\max }^{1 /(1-k)}\right)}{T_{1}\left(x_{\max }-1\right)-T_{4}\left(1-1 / x_{\max }\right)+(k-1) T_{4}\left(1-1 / r_{c}\right)}\right]^{(1-k) / k}
$$

Foi verificado computacionalmente que o resultado da Eq. (70) resulta em um máximo da Eq. (59). A Eq. (70) deve ser resolvida numericamente para a obtenção do valor de $x_{\max }$. Com o valor de $x_{\max }$, podem ser calculados valores de $\left(\dot{W}_{\text {cillo,Dual }}\right)_{\max }, r_{\text {max,Dual }}, P M E_{\text {max,Dual }}$ e $\eta_{\text {max,Dual }}$, respectivamente através das seguintes expressões:

$$
\begin{gathered}
\left(\dot{W}_{\text {ciclo, Dual }}\right)_{\max }=\dot{m} c_{v}\left[T_{3}\left(1-1 / x_{\max }\right)-T_{1}\left(x_{\max }-1\right)\right] \\
r_{\text {max,Dual }}=\left(x_{\max }\right)^{1 /(1-k)} \\
P M E_{\text {max }, \text { Dual }}=\frac{\dot{m} c_{v}}{V_{1}} \times \frac{\left[T_{4}\left(1-r_{c}^{k-1} / x_{\max }\right)+(k-1) T_{4}\left(1-1 / r_{c}\right)-T_{1}\left(x_{\max }-1\right)\right]}{1-x_{\max }^{1 / 1-k)}} \\
\eta_{\max , \text { Dual }}=\frac{T_{3}\left(1-1 / x_{\max }\right)-T_{2}\left(x_{\max }-1\right)}{T_{3}-T_{1} x_{\max }}
\end{gathered}
$$

\section{ANÁLISE COMPARATIVA ENTRE OTIMIZAÇÕES}

Limitações de ordem material na construção de motores ditam o máximo limite permissível de temperatura da substância de trabalho, $T_{3}$ e $T_{4}$. Além disso, devido a ineficiência do processo de combustão, essas temperaturas máximas devem ser menores que a temperatura adiabática de chama correspondente ao combustível utilizado. Assim, 
para motores reais, existe uma faixa de temperaturas $T_{3}$ e $T_{4}$ que pode ser encontrada. A título de ilustração, foi considerado que $T_{3}=T_{4}$ com uma faixa de variação entre $2000 \mathrm{~K}$ e $3000 \mathrm{~K}$ para comparação entre os diversos valores de $r, \dot{W}_{\text {ciclo }}$, PME e $\eta$ para as duas otimizações propostas e para os três ciclos considerados. Para todos os cálculos foi considerado que $T_{1}=300 \mathrm{~K}, \quad \dot{m}=1 \mathrm{~kg} / \mathrm{s}, \quad c_{v}=0,7165 \mathrm{~kJ} / \mathrm{kg} . \mathrm{K}, \quad c_{p}=1,0035 \mathrm{~kJ} / \mathrm{kg} . \mathrm{K}, \quad k=1,4, \quad r_{c}=1,5, \quad r_{p}=1,2 \quad \mathrm{e}$ $V_{1}=0,8 \mathrm{~m}^{3}$.

De acordo com os valores constantes considerados anteriormente e a formulação apresentada nesse trabalho, foram calculados as variações dos valores dos quatro parâmetros $r, \dot{W}_{\text {ciclo }}$, PME e $\eta$ para as duas otimizações propostas e para os três ciclos considerados utilizando intervalos de $10 \mathrm{~K}$ dentro da faixa de temperaturas entre $2000 \mathrm{~K}$ e $3000 \mathrm{~K}$. Os resultados da formulação apresentada podem ser vistos na Fig. 4. Essa figura mostra simultaneamente soluções para: $r_{o t m}$ e $r_{\max }$ em função de $T_{3} ;\left(\dot{W}_{\text {ciclo }}\right)_{o t m}$ e $\left(\dot{W}_{\text {ciclo }}\right)_{\max }$ em função de $T_{3} ; P M E_{\text {otm }}$ e $P M E_{\max }$ em função de $T_{3}$; e $\eta_{\text {otm }}$ e $\eta_{\max }$ em função de $T_{3}$. Como pode ser visto através do resultados, as relações de compressão necessárias para a obtenção da potência líquida de saída ótima são substancialmente maiores que aquelas necessárias para a obtenção da PME máxima. Além disso, a eficiência térmica para o caso potência líquida de saída otimizada é substancialmente maior que aquela para o caso da potência líquida de saída máxima para toda a faixa de temperatura considerada.

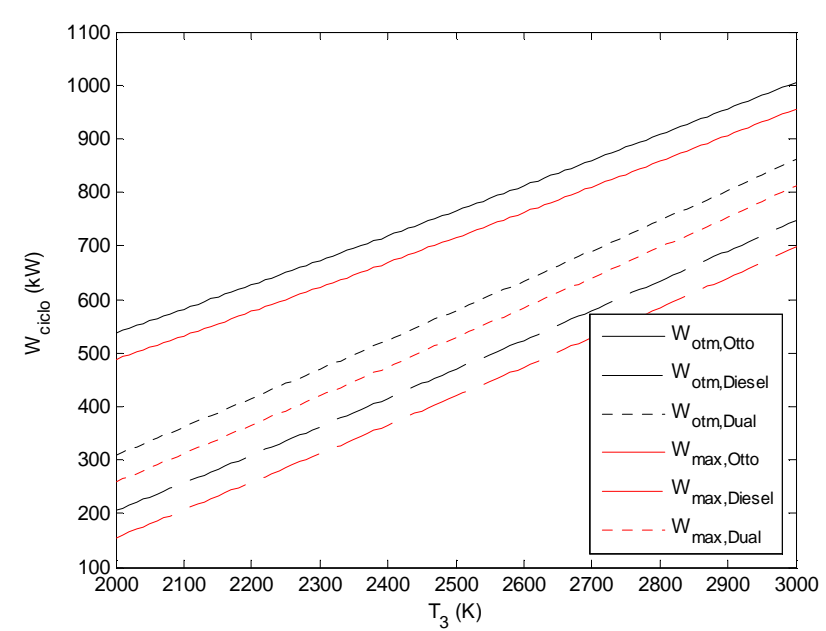

(a)

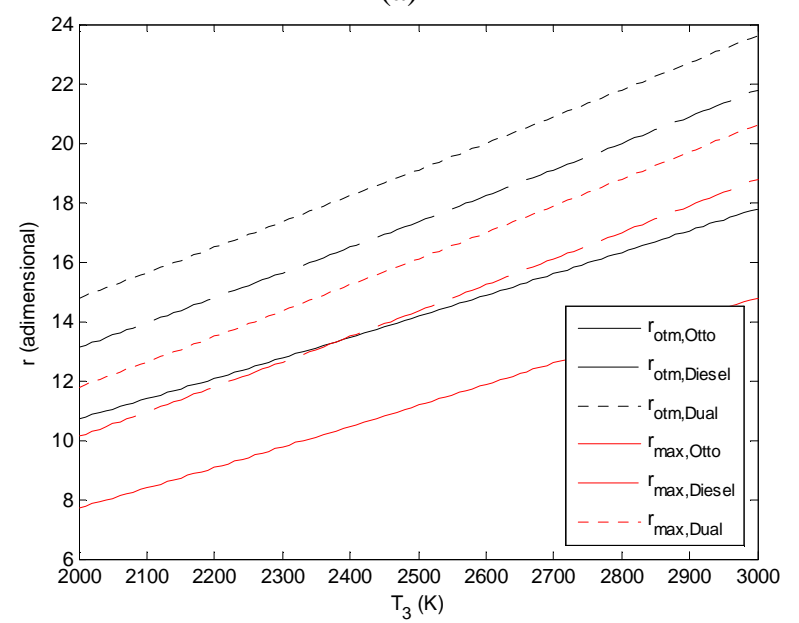

(a)

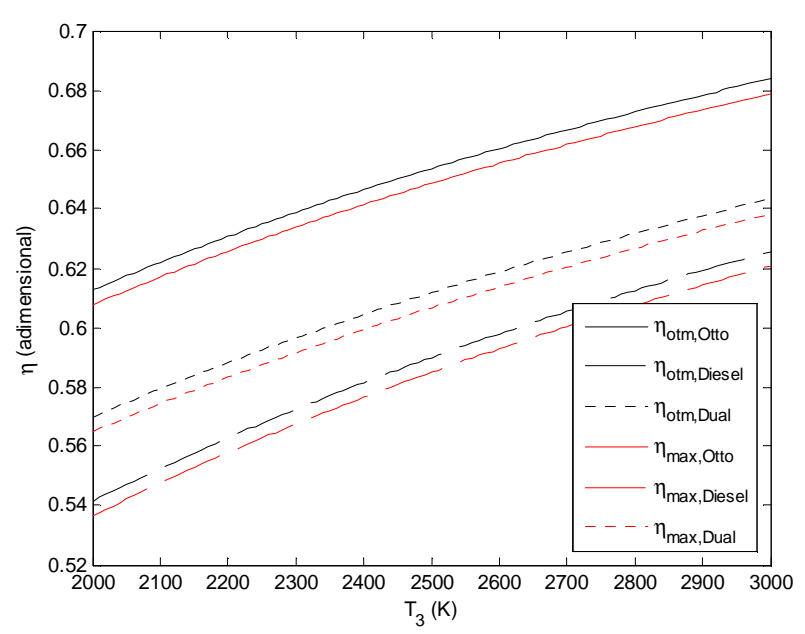

(b)

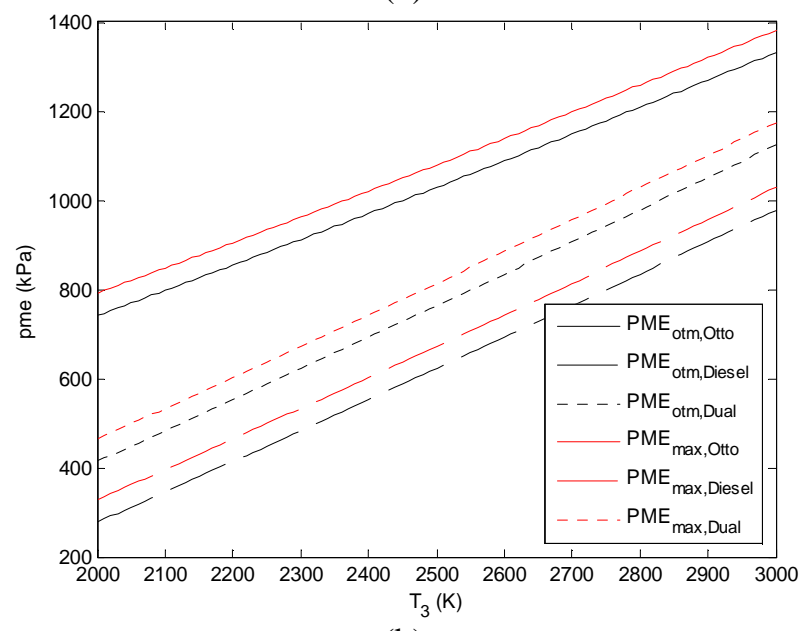

(b)

Figura 4 - Variáveis do ciclo em função da variação da temperatura $T_{3}$.

\section{CONCLUSÕES}

Nesse trabalho foi realizada uma otimização da potência líquida de saída e da pressão média efetiva dos ciclos de ar-padrão Otto, Diesel e Dual. Os resultados obtidos podem ser utilizados como critério para utilização em cálculos de desempenho e adequação de motores Otto, Diesel e Dual. 


\title{
REFERÊNCIAS
}

Chen, L., Zeng, F., Sun, F., Wu, C. 1996. Heat transfer effects on the net work and/or power ad functions of efficiency for air-standards Diesel cycles. Energy, 21(12), 1201-1205.

Chen, L., Wu, C., Sun, F., Cao, S. 1998. Heat transfer effects on the net work output and efficiency characteristics for an air-standard Otto cycle. Energy Conversion and Management, 39(7), 643-648.

Moran, M. J., Shapiro, H. N., Boettner, D. D., Bailey, M. B. 2013. Princípios de Termodinâmica para Engenharia. Rio de Janeiro, Brazil: Ed. LTC. 819 p.

\section{RESPONSABILIDADE AUTORAL}

"Os autores são os únicos responsáveis pelo conteúdo deste trabalho".

\section{OPTIMIZATION OF AIR STANDARD AIR CYCLES OTTO, DIESEL AND DUAL WITH RESPECT TO NET POWER OUTPUT AND MEAN EFFECTIVE PRESSURE}

\author{
Santiago del Rio Oliveira, santiago@feb.unesp.br ${ }^{1}$ \\ Vicente Luiz Scalon, scalon@ feb.unesp.br ${ }^{1}$ \\ ${ }^{1}$ Universidade Estadual Paulista Júlio de Mesquita Filho/UNESP - Departamento de Engenharia Mecânica - Av. Eng. \\ Luiz Edmundo C. Coube, Bairro Vargem Limpa, CEP 17033-360, Bauru/SP.
}

\begin{abstract}
In this work the endoreversible air-standard cycles Otto, Diesel and Dual are optimized with respect to net output power and mean effective pressure. The endoreversible cycle is one in which a heat supply process from the combustion process and heat rejection to the environment are the only irreversible processes in the cycle. Expressions for the optimized net power output and for the optimized mean effective pressure for each engine cycle is obtained and is then carried out a comparative analysis of the results. These results can be used as an additional criteria to use in evaluating the adequacy and performance of internal combustion engines that operate on the Otto, Diesel and Dual cycles.
\end{abstract}

Keywords: Otto, Diesel, Dual, optimization. 